\title{
Effect of Scatter Correction When Comparing Attenuation Maps: Application to Brain PET/MR
}

\author{
Ninon Burgos, Kris Thielemans, M. Jorge Cardoso, Pawel Markiewicz, Jieqing Jiao, John Dickson, \\ John S. Duncan, David Atkinson, Simon R. Arridge, Brian F. Hutton, and Sébastien Ourselin
}

\begin{abstract}
In PET imaging, attenuation and scatter corrections are an essential requirement to accurately quantify the radionuclide uptake. In the context of PET/MR scanners, obtaining the attenuation information can be challenging. Various authors have quantified the effect of an imprecise attenuation map on the reconstructed PET image but its influence on scatter correction has usually been ignored.

In this paper, we investigate the effects of imperfect attenuation maps ( $\mu$ maps) on the scatter correction in a simulation setting. We focused our study on three $\mu$ maps: the reference $\mu$ map derived from a CT image, and two MR-based methods. Two scatter estimation strategies were implemented: a $\mu$ map-specific scatter estimation and an ideal scatter estimation relying only on the reference CT $\mu$ map. The scatter estimation used the Single Scatter Simulation algorithm with tail-fitting.

The results show that, for FDG brain PET, regardless of the $\mu$ map used in the reconstruction, the difference on PET images between $\mu$ map-specific and ideal scatter estimations is small (less than 1\%). More importantly, the relative error between attenuation correction methods does not change depending on the scatter estimation method included in the simulation and reconstruction process. This means that the effect of errors in the $\mu$ map on the PET image is dominated by the attenuation correction, while the scatter estimate is relatively unaffected. Therefore, while scatter correction improves reconstruction accuracy, it is unnecessary to include scatter in the simulation when comparing different attenuation correction methods for brain PET/MR.
\end{abstract}

\section{INTRODUCTION}

$\mathbf{I}$ N Positron Emission Tomography (PET) imaging, correcting for photon attenuation is an essential requirement to accurately quantify the radionuclide uptake. For PET/MR imaging, in the absence of a transmission source or Computed Tomography (CT) image, alternative Magnetic Resonance (MR)based methods are being developed. Due to the challenge of delineating bone in MR images, these attenuation maps can be inaccurate and lead in some cases to a strong spatial bias of the

Manuscript received May 8, 2014. This work was supported by an IMPACT studentship funded jointly by Siemens and the UCL FES. The Dementia Research Centre is an Alzheimer's Research UK Coordinating Centre and has also received equipment funded by Alzheimer's Research UK and Brain Research Trust. Funding was received from the EPSRC (EP/H046410/1, EP/J020990/1, EP/K005278), the MRC (MR/J01107X/1), the EU-FP7 project VPH-DARE@IT (FP7-ICT-2011-9-601055) and the NIHR Biomedical Research Unit (Dementia) at UCL. This work was also supported by researchers at the NIHR UCLH BRC (including the High Impact Initiative).

N. Burgos, P. Markiewicz and S.R. Arridge are with the Centre for Medical Image Computing, UCL, UK (email: n.burgos.12@ucl.ac.uk). K. Thielemans, J. Dickson and B.F. Hutton are with the Institute of Nuclear Medicine, UCL, UK. M.J. Cardoso, and S. Ourselin are with Centre for Medical Image Computing and the Dementia Research Centre, UCL, UK. J.S. Duncan is with Department of Clinical and Experimental Epilepsy, UCL IoN, London, UK. D. Atkinson is with the Centre for Medical Imaging, UCL, UK.
PET activity, as shown for instance in [1]. Scatter correction is another key component for the quantitative analysis of PET images. Various authors have quantified the effect of an imprecise attenuation map on the final reconstructed PET image, but its influence on scatter correction has usually been ignored.

Compton scattering is the main interaction of $511-\mathrm{keV}$ photons, emitted following annihilation between a positron and an electron, with tissue. Compton effect leads to two phenomena: attenuation and scatter, both a consequence of the same physical process. Photons undergoing Compton scattering will deviate from their original path. As a result, they might not be detected (attenuation) or they might still be detected but their annihilation site will be wrongly located (scatter). In order to accurately quantify the radionuclide uptake, the emission data need to be corrected for both attenuation and scatter.

In combined PET/CT systems, the attenuation information is derived from a CT image as the Hounsfield unit scale is a linear transformation of the linear attenuation coefficients. Regarding PET/MR scanners, as MRI image intensities do not reflect the electron densities, new methods have to be developed [2]. These methods can be classified in two main categories: segmentation and registration-based approaches. The segmentation-based strategy consists of assigning uniform linear attenuation coefficients to tissue classes obtained by segmenting an MRI image. In registration-based methods, an attenuation map template, derived from pre-acquired CT or transmission images, is deformed to match the patient's anatomy.

Several methods exist to correct for scatter [3]. They include using multiple energy windows, fitting a curve to data outside the patient, and calculating scatter from the emission and attenuation data using a model or Monte Carlo simulation.

In this paper, we propose to investigate, using simulations, the effect of imperfect attenuation maps ( $\mu$ maps) on the scatter correction. We focus on three $\mu$ maps: a $\mu$ map derived from a CT image, considered as the reference, and two MRbased methods: a segmentation-based approach [4] and a registration-based approach developed by [5].

\section{METHOD}

\section{A. MR-based Attenuation Correction}

1) Segmentation-based Approach: The UTE-based method, a prototype version implemented on the first software versions of the Siemens Biograph mMR hybrid PET/MR scanners, is based on the segmentation of a Ultrashort-Echo-Time (UTE) 


\author{
Simulation: \\ Input: True emission image $E_{T}$, true $\mu$ map $\mu_{T}$ \\ Output: Simulated PET sinogram $S$ \\ 1: Forward project $E_{T}$ \\ 2: Simulate attenuation from $\mu_{T}$ \\ 3: Simulate scatter with SSS from $\mu_{T}$ and $E_{T}$ \\ 4: Combine to obtain simulated PET sinogram $S$ \\ Reconstruction: \\ Input: Simulated PET sinogram $S$ and other $\mu$ map $\mu_{O}$ \\ Output: Reconstructed PET image \\ 1: Estimate attenuation from $\mu_{O}$ \\ 2: Estimate scatter from $S$ and $\mu_{O}$ \\ 3: Run OSEM using $S$ and the output of 1 and 2
}

Fig. 1. PET simulation and reconstruction process with $\mu$ map-specific scatter estimation.

MRI sequence which enables the differentiation of three tissue classes (bone, air and soft tissue). Predefined attenuation coefficients are then assigned to each tissue class.

2) Registration-based Approach: The multi-atlas CT synthesis method developed by Burgos et al. [5] relies on a preacquired set of aligned MRI/CT image pairs from multiple subjects forming an MRI-CT database. To generate the CT from a target MRI, each MRI image from the database is deformed to the target MRI using affine followed by non-rigid registration. The CT images in the database are then mapped using the same transformation to the target MRI image. A local image similarity measure between the target MRI and the set of registered MRIs from the database is used as a surrogate of the underlying morphological similarity, under the assumption that if two MRIs are similar at a certain spatial location, the two CTs will also be similar at this location. Finally, the set of registered CTs is fused using a voxel-wise weighting scheme, generating a pseudo CT (pCT). To obtain the $\mu$ map, the CT values expressed in $\mathrm{HU}$ are converted to linear attenuation coefficients in $\mathrm{cm}^{-1}$ by a piecewise linear transformation.

\section{B. Scatter Correction}

In this work, scatter was estimated using a Single Scatter Simulation (SSS) algorithm [6] and scaled using a tail-fitting strategy, as implemented in STIR [7]. The SSS algorithm consists of using the emission and attenuation information to model single Compton scattering. The estimated scatter is then scaled to fit the tails of the sinogram, obtained from the attenuation map, where it is assumed that the signal is only coming from scattered events. Finally, the scatter estimation and image reconstruction steps are combined in an iterative process as follows:

1) Reconstruction of an initial estimate of the emission data.

2) Single scatter estimation using the SSS algorithm.

3) Tail-fitting.

4) Correction of emission data: the estimated scatter is subtracted from the emission data.

5) Reconstruction of the emission data, and back to step 2 .
Simulation:

Input: True emission image $E_{T}$, true $\mu$ map $\mu_{T}$ Output: Simulated PET sinogram $S^{\prime}$

1: Forward project $E_{T}$

2: Simulate attenuation from $\mu_{T}$

3: Combine to obtain simulated PET sinogram $S^{\prime}$

\section{Reconstruction:}

Input: Simulated PET sinogram $S^{\prime}$ and other $\mu$ map $\mu_{O}$ Output: Reconstructed PET image

1: Estimate attenuation from $\mu_{O}$

2: Run OSEM using $S^{\prime}$ and the output of 1

Fig. 2. PET simulation and reconstruction process with ideal scatter estimation.

\section{PET Simulation and Reconstruction}

PET images were reconstructed with different $\mu$ maps following a projection/reconstruction technique similar to [5]. To assess the effect of imperfect $\mu$ maps on the scatter correction, a reference PET image with accurate scatter correction is required. The ideal scatter correction is obtained from the PET/CT scanner and relies on the reference CT $\mu$ map. We detail a first simulation and reconstruction process where the scatter is estimated from the different $\mu$ maps and a second process where the scatter is estimated from the reference CT $\mu$ map in the PET/CT scanner.

1) Process with $\mu$ map-specific Scatter Correction: As input for the simulations, we used the CT and reconstructed PET images provided by a PET/CT scanner. The PET simulation and reconstruction process with $\mu$ map-specific scatter correction $\left(\mathrm{SC}_{\mu \text { map }}\right)$ is detailed in Fig. 1. The non-corrected PET sinogram $S$, equivalent to the measured data, was obtained by removing the attenuation and scatter corrections from the original simulated PET sinogram. PET images were then reconstructed using the $\mathrm{CT}$, pCT or UTE $\mu$ map to correct for attenuation and scatter, i.e. the scatter was computed from the PET sinogram and the estimated $\mu$ map.

Image reconstruction was performed using an Ordered Subsets Expectation Maximisation (OSEM) algorithm with 3 iterations of 21 subsets using the STIR package [7]. The scatter estimate was incorporated as a background term in the forward model, i.e. in the denominator of the OSEM update. Effects of PSF and randoms were not included and post-reconstruction smoothing was not applied.

2) Process with Ideal Scatter Correction: For the second strategy, the same process was followed but without scatter in both the simulation and reconstruction. These images can be interpreted as being reconstructed with perfect scatter elimination, but an approximate attenuation map. The PET simulation and reconstruction process with ideal scatter correction $\left(\mathrm{SC}_{\text {ideal }}\right)$ is summarised in Fig. 2 . The simulated PET sinogram $S^{\prime}$ was produced by removing only the attenuation correction from the original PET sinogram. PET images were then reconstructed using the different $\mu$ maps to correct for attenuation only. 
TABLE I

FOR 41 SUBJECTS: AVERAGE AND SD OF THE RMAE AND RME BETWEEN $\mathrm{SC}_{\mu \text { map }}$ AND $\mathrm{SC}_{\text {ideal }}$ PET IMAGES, FOR IMAGES RECONSTRUCTED WITH 3 ATTENUATION MAPS, IN THE FULL HEAD AND BRAIN REGIONS.

\begin{tabular}{|c|c|c|c|c|c|c|c|}
\cline { 3 - 8 } \multicolumn{2}{c|}{} & \multicolumn{3}{c|}{ Head } & \multicolumn{3}{c|}{ Brain } \\
\cline { 3 - 8 } \multicolumn{2}{c|}{} & CT & pCT & UTE & CT & pCT & UTE \\
\hline \multirow{2}{*}{ rMAE (\%) } & Average & 1.27 & 1.37 & 1.45 & 0.81 & 0.85 & 0.88 \\
\cline { 2 - 8 } & SD & 0.14 & 0.15 & 0.16 & 0.07 & 0.08 & 0.08 \\
\hline \multirow{2}{*}{ rME (\%) } & Average & -0.92 & -0.91 & -0.81 & -0.58 & -0.59 & -0.54 \\
\cline { 2 - 8 } & SD & 0.12 & 0.15 & 0.14 & 0.06 & 0.08 & 0.08 \\
\hline
\end{tabular}

\section{RESULTS}

Data: 41 brain T1-w MRIs, CTs, UTE-derived $\mu$ maps and reconstructed PETs were used. The T1-w MRIs (3.0 T; TE/TR/TI, $2.63 \mathrm{~ms} / 1700 \mathrm{~ms} / 900 \mathrm{~ms}$; flip angle $9^{\circ}$; voxel size $0.53 \times 0.53$ $\times 1.1 \mathrm{~mm}^{3}$ ) and UTE $\mu$ maps (voxel size $1.562 \times 1.562 \times$ $1.562 \mathrm{~mm}^{3}$ ) were acquired on a Siemens Biograph mMR hybrid PET/MR scanner; the CTs (voxel size $0.586 \times 0.586 \times 1.25 \mathrm{~mm}^{3}$, $120 \mathrm{kVp}, 300 \mathrm{~mA}$ ) and reconstructed PETs (radiopharmaceutical: FDG; voxel size $1.953 \times 1.953 \times 3.27 \mathrm{~mm}^{3}$ ) on a GE Discovery ST PET/CT scanner.

For the three $\mu$ maps considered (CT, pCT and UTE-based), PET images were obtained using the simulation and reconstruction processes with both the $\mu$ map-specific and ideal scatter corrections.

\section{A. Effect of Wrong $\mu$ map on Scatter Correction}

In a first instance, we analysed, for the three $\mu$ maps, the difference between PET images simulated and reconstructed following the ideal scatter estimation process and PET images simulated and reconstructed following the $\mu$ map-specific scatter process. To do so, the relative mean absolute error, defined as $\mathrm{rMAE}=100 * \sum_{\vec{v}}\left|I_{\vec{v}}-J_{\vec{v}}\right| / \sum_{\vec{v}} J_{\vec{v}}$, and the relative mean error, $\mathrm{rME}=100 * \sum_{\vec{v}}\left(I_{\vec{v}}-J_{\vec{v}}\right) / \sum_{\vec{v}} J_{\vec{v}}$, were computed between the $\mathrm{SC}_{\mu \text { map }} \operatorname{PET}(I)$ and $\mathrm{SC}_{\text {ideal }}$ PET $(J)$ images. Results obtained in the head and brain regions are shown in Table I. Regardless of the $\mu$ map used, the rMAE between $\mathrm{SC}_{\mu \text { map }}$ and $\mathrm{SC}_{\text {ideal }}$ PET images is small (less than $1 \%$ in the brain). Examples of $\mu$ maps and PET images are presented in Fig. 3 for a representative subject.

For this representative subject, profiles of the true scatter, obtained from the $\mathrm{SC}_{\mu \text { map }}$ simulation, and of the scatter estimates, obtained from the simulated sinogram $S$ and the CT, pCT and UTE $\mu$ maps, were analysed (Fig. 4). We note that the difference between true and estimated scatter, regardless of the $\mu$ map, is small, which agrees with the results obtained when comparing $\mathrm{SC}_{\text {ideal }}$ and $\mathrm{SC}_{\mu \text { map }}$ PET images.

To localise the effects of the attenuation and scatter corrections, the PET images from the 41 subjects were mapped to a common space via a CT-based groupwise registration [8]. Fig. 5 presents the difference, averaged across the 41 subjects, between $\mathrm{SC}_{\mu \text { map }}$ and $\mathrm{SC}_{\text {ideal }}$ PET images, for the $\mathrm{CT}$, $\mathrm{pCT}$ and UTE $\mu$ maps. These images confirmed the results presented in Table I.

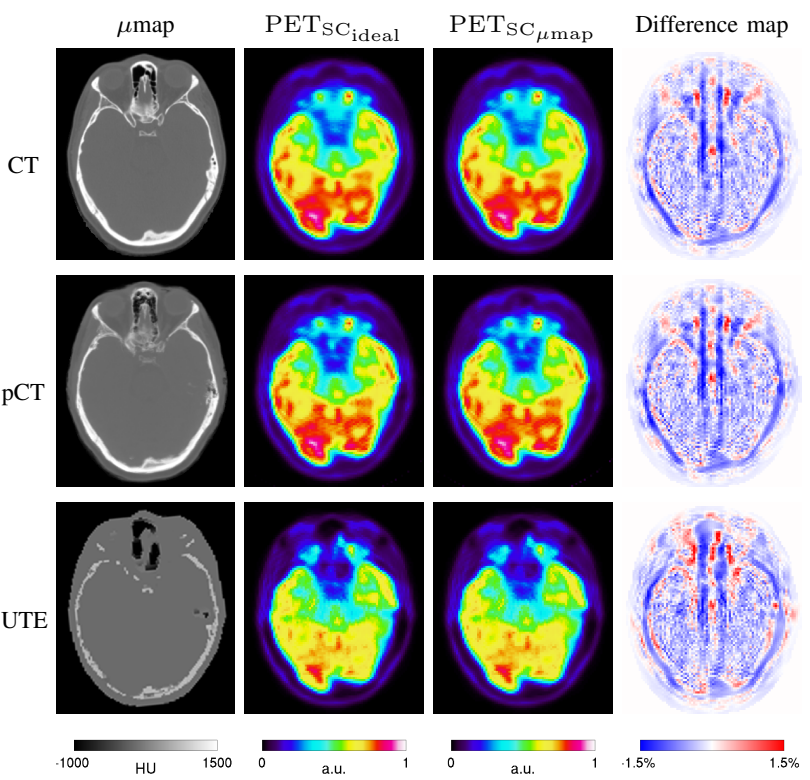

Fig. 3. Example of $\mu$ map, PET image obtained with ideal $\left(\mathrm{SC}_{\text {ideal }}\right)$ and $\mu$ map-specific $\left(\mathrm{SC}_{\mu \text { map }}\right)$ scatter estimation and difference between $\mathrm{SC}_{\text {ideal }}$ and $\mathrm{SC}_{\mu \text { map }}$ PETs $\left(\mathrm{PET}_{\mathrm{SC}_{\text {ideal }}}-\mathrm{PET}_{\mathrm{SC}_{\mu \mathrm{map}}}\right)$, for the ground truth $\mathrm{CT}$ $\mu$ map (top), the pseudo CT $\mu$ map (middle) and the UTE $\mu$ map (bottom).
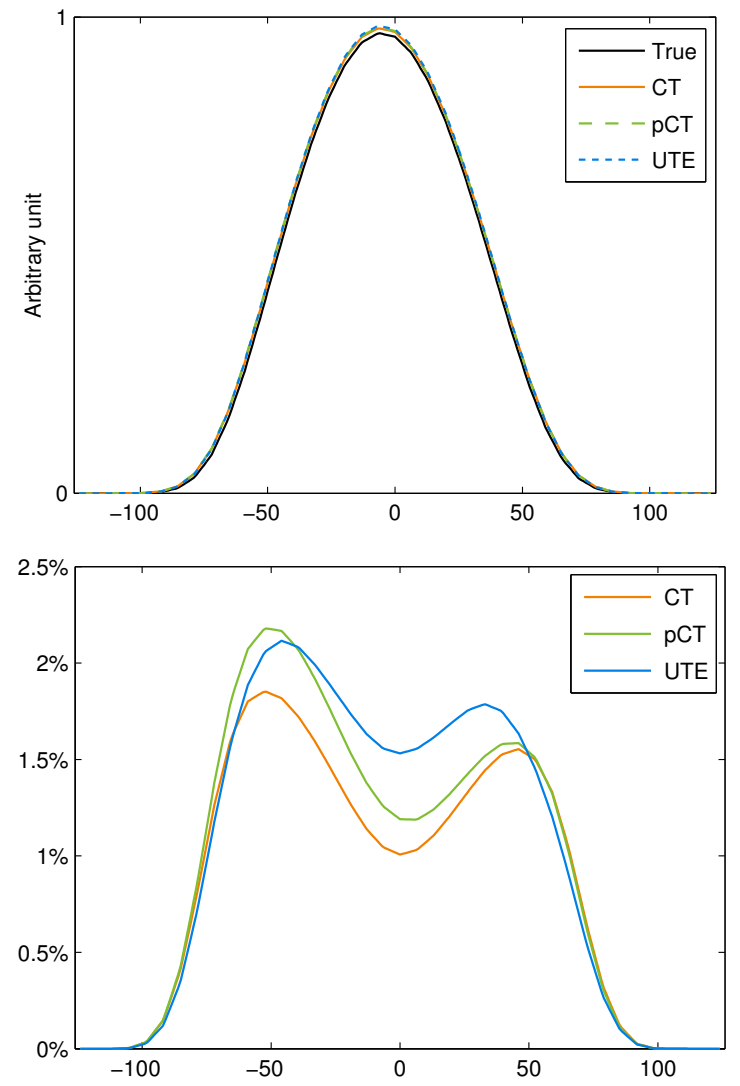

Fig. 4. Profiles of the true scatter obtained from the $\mathrm{SC}_{\mu \text { map }}$ simulation and of the scatter estimated with three $\mu$ maps (top); difference between estimated and true scatter (bottom). 
(a)
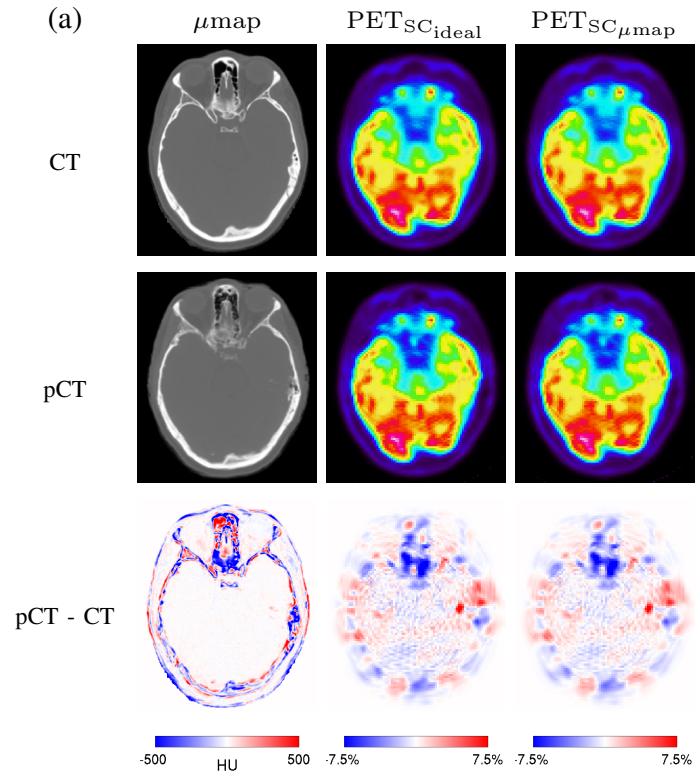

(b)
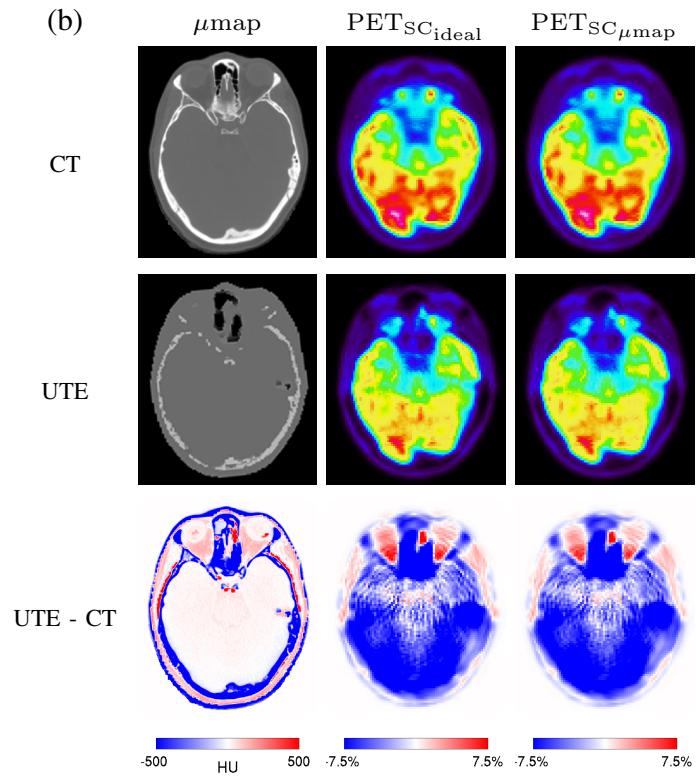

Fig. 6. Examples of MR-based $\mu$ maps (pCT (a) and UTE (b)), PET images corrected for attenuation using the CT $\mu$ map, PET images corrected for attenuation using the MR-based $\mu$ map and differences between CT and MR-based PET images, for the $\mu$ map-specific scatter estimation and the ideal scatter estimation.

CT

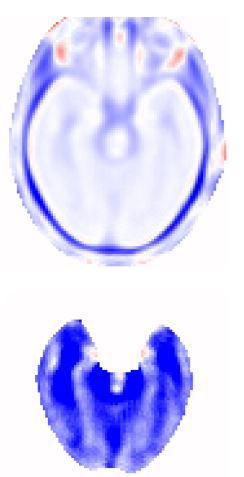

pseudo CT
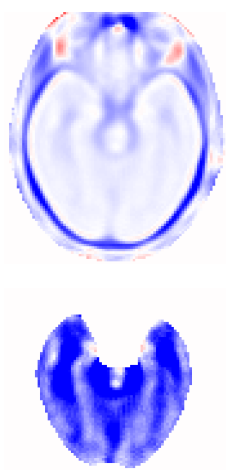

UTE

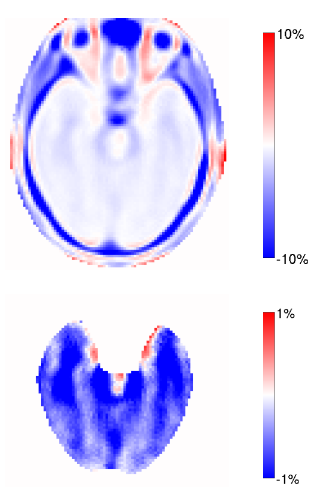

Fig. 5. Difference, averaged over 41 subjects, between the PETs reconstructed with the ideal scatter correction and the PETs reconstructed with the $\mu$ mapspecific scatter correction, for three attenuation maps.

\section{B. Effect of Including Scatter when Comparing $\mu$ maps}

In a second instance, we studied the effect of different scatter estimation processes when comparing MR-based $\mu$ maps to the reference CT $\mu$ map. The rMAE and rME were calculated for every subject between the gold standard PET $(J)$, reconstructed using the CT $\mu$ map, and the PET images reconstructed using the pCT and UTE $\mu$ maps $(I)$, for the head and brain regions. Results are shown in Table II. Regardless of the scatter correction, the average rMAE obtained in the brain using the $\mathrm{pCT} \mu$ map is 4 times smaller than the error obtained using the UTE $\mu$ map.

For the subject shown in Fig. 3, we computed the difference between the CT and UTE PET images and between the CT and pCT PET images for both scatter estimation processes. The images are displayed in Fig. 6.

Using the groupwise analysis described in the previous
TABLE II

FOR 41 SUBJECTS: AVERAGE AND SD OF THE RMAE AND RME BETWEEN THE GOLD STANDARD CT PET AND BOTH THE PSEUDO CT AND UTE PETS, FOR THE $\mu$ MAP-SPECIFIC AND IDEAL SCATTER PROCESSES, IN THE FULL HEAD AND BRAIN REGIONS.

\begin{tabular}{|c|c|c|c|c|c|c|c|c|c|}
\cline { 2 - 11 } \multicolumn{2}{c|}{} & \multicolumn{4}{c|}{ Head } & \multicolumn{4}{c|}{ Brain } \\
\cline { 2 - 11 } \multicolumn{2}{c|}{} & $\mathrm{SC}_{\mu \text { map }}$ & \multicolumn{2}{c|}{$\mathrm{SC}_{\text {ideal }}$} & \multicolumn{2}{|c|}{$\mathrm{SC}_{\mu \text { map }}$} & \multicolumn{2}{|c|}{$\mathrm{SC}_{\text {ideal }}$} \\
\cline { 2 - 10 } \multicolumn{2}{c|}{} & pCT & UTE & pCT & UTE & pCT & UTE & pCT & UTE \\
\hline \multirow{2}{*}{ rMAE (\%) } & Average & 5.60 & 14.67 & 5.77 & 14.73 & 2.89 & 11.86 & 2.95 & 11.85 \\
\cline { 2 - 11 } & SD & 1.69 & 1.98 & 1.68 & 1.95 & 0.91 & 2.09 & 0.88 & 2.08 \\
\hline \multirow{2}{*}{ rME (\%) } & Average & -1.07 & -13.71 & -1.09 & -13.61 & 0.15 & -11.78 & 0.14 & -11.74 \\
\cline { 2 - 10 } & SD & 2.58 & 2.15 & 2.52 & 2.14 & 2.12 & 2.13 & 2.10 & 2.13 \\
\hline
\end{tabular}

pseudo CT

UTE

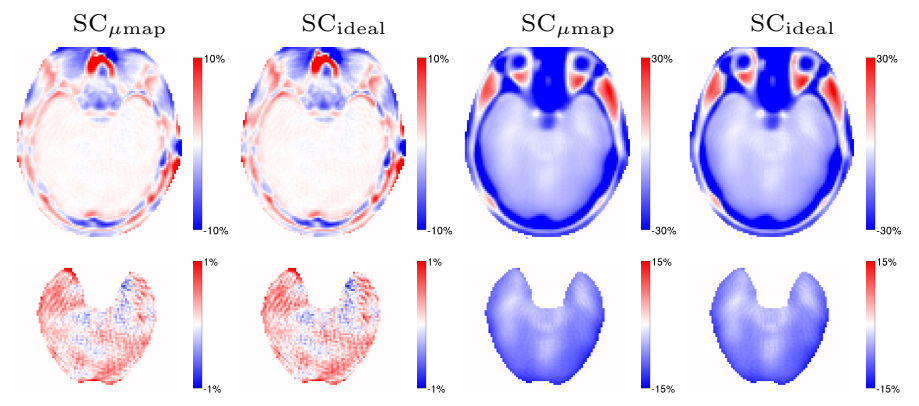

Fig. 7. Average over 41 subjects of the difference between the PETs reconstructed with the ground truth CT $\mu$ map and the PETs reconstructed with the pCT $\mu$ map (left) or the UTE $\mu$ map (right), for both $\mu$ map-specific and ideal scatter estimation, in the whole head (top) and brain region (bottom).

section, averaged difference maps were computed between the gold standard PETs and the PETs reconstructed with the pCT and UTE $\mu$ maps, for both scatter estimation processes (Fig 7). As expected, regardless of the scatter estimation method, the difference between the gold standard and pCT PETs is lower than between the gold standard and UTE PETs, as the pCTbased attenuation provides more realistic $\mu$ maps. 


\section{CONCLUSION}

This paper presents an analysis of the effects of imperfect attenuation maps on the scatter correction. Regardless of the $\mu$ map used in the reconstruction, the difference on PET images between $\mu$ map-specific and ideal scatter estimation is small, particularly in the brain region (maximum average difference of $0.88 \%$ for the worst method). More importantly, the relative error between attenuation correction methods does not change depending on the scatter estimation method included in the simulation and reconstruction process. This means that the effect of errors in the $\mu$ map on the PET image is dominated by the attenuation correction, while the scatter estimate is relatively unaffected. Therefore, while scatter correction improves reconstruction accuracy, it is unnecessary to include scatter in the simulation when comparing different attenuation correction methods.

The current analysis used FDG brain images. The conclusions could be different for tracers such as F-DOPA with uptake in specific regions, or for the thorax.

\section{REFERENCES}

[1] J. C. Dickson, C. O'Meara, and A. Barnes, "A comparison of CT- and MR-based attenuation correction in neurological PET." European Journal of Nuclear Medicine and Molecular Imaging, 2014.

[2] I. Bezrukov, F. Mantlik, H. Schmidt, B. Schölkopf, and B. J. Pichler, "MR-Based PET Attenuation Correction for PET/MR Imaging," Seminars in Nuclear Medicine, vol. 43, no. 1, pp. 45-59, 2013.

[3] H. Zaidi and K. Koral, "Scatter modelling and compensation in emission tomography," European Journal of Nuclear Medicine and Molecular Imaging, vol. 31, no. 5, pp. 761-782, 2004.

[4] V. Keereman, Y. Fierens, T. Broux, Y. De Deene, M. Lonneux, and S. Vandenberghe, "MRI-based attenuation correction for PET/MRI using ultrashort echo time sequences." Journal of Nuclear Medicine, vol. 51, no. 5, pp. 812-8, 2010.

[5] N. Burgos, M. J. Cardoso, K. Thielemans, M. Modat, S. Pedemonte, J. Dickson, A. Barnes, R. Ahmed, C. J. Mahoney, J. M. Schott, J. S. Duncan, D. Atkinson, S. R. Arridge, B. F. Hutton, and S. Ourselin, "Attenuation Correction Synthesis for Hybrid PET-MR Scanners: Application to Brain Studies," IEEE Transactions on Medical Imaging, 2014.

[6] C. Watson, "New, Faster, Image-Based Scatter Correctionfor 3D PET." IEEE Nuclear Science Symposium and Medical Imaging Conference, vol. 47 , no. 4 , pp. $1587-1594,2000$.

[7] K. Thielemans, C. Tsoumpas, S. Mustafovic, T. Beisel, P. Aguiar, N. Dikaios, and M. W. Jacobson, "STIR: software for tomographic image reconstruction release 2." Physics in Medicine and Biology, vol. 57, no. 4 pp. 867-83, 2012.

[8] T. Rohlfing, R. Brandt, J. Maurer, C.R., and R. Menzel, "Bee brains, Bsplines and computational democracy: generating an average shape atlas," IEEE Workshop on Mathematical Methods in Biomedical Image Analysis, pp. 187-194, 2001. 